\title{
Continuous Welded Rail in Permafrost Regions Deformation Analysis of Qinghai-Tibet railway
}

\author{
Mu Hang ${ }^{1, a}$ \\ ${ }^{1}$ School of Civil Engineering, Lanzhou Jiaotong University, Lanzhou China 730070 \\ amuhang@mail.Izjtu.cn
}

Keywords : CWR ; deformation ; settlemen.

\begin{abstract}
Domestic and foreign research on the stability of seamless track plane frame have relatively mature achievement. And it can able to accurately calculate long rail temperature inside force under the condition of different parameters. But there are a few literature reported about under the condition of vertical settlement of track stability analysis. The paper study about under the condition of vertical settlement of track deformation analysis. Analysis of track displacement distribution and the law of development under the condition of embankment settlement diseases based on nonlinear finite element method. Research indicates that rail vertical displacement and roadbed subsidence has the linear relationship in the range of track bed resistance to be effective. When the roadbed arch and sink alternately that will be a significant increase in rail displacement.
\end{abstract}

\section{Introduction}

The total length of Golmud-Lhasa section of the Qinghai-Tibet Railway is $1142 \mathrm{~km}$, including $960 \mathrm{~km}$ for above $4000 \mathrm{~m}$ high, $546 \mathrm{~km}$ for permafrost section and $593 \mathrm{~km}$ long seasonal frozen soil section. ${ }^{[1]}$ Due to the repeated frost heaving and thawing of the subsoil in permafrost regions, the embankment is easy to get settlement or deformation ${ }^{[2][3]}$. At present, the theoretical research methods of the stability of CWR are mainly analyzed by the analysis method of the stability of the jointless track, the finite element model of the jointless track and the dynamic stability analysis of the jointless track ${ }^{[4]}$. In the study mentioned above, the stability of CWR caused by different initial irregularities, different radius of curvature and different fastener resistance is considered in the general subgrade section ${ }^{[5][6]}$. However, the stability analysis of CWR under vertical settlement is still lacking in depth. In this paper, nonlinear finite element method is used to analyze the influence of vertical settlement on the thermal stress of CWR rail, and put forward permafrost zone pavement and maintenance Theoretical Basis of Jointless Track.

\section{Mechanical model}

The beam is used to simulate the long rail, and the restraint system is simulated by longitudinal and lateral spring restraints. The rotation restraint spring is used to simulate the torsion constraint of the fastener on the long rail. Assuming an initial asymmetric distribution of the long rail, the model can be reduced to half the actual state ${ }^{[7]}$. On the basis of the literature [7], considering both the long-rail torsional displacement and the long-rail longitudinal and lateral displacement of the discrete [8][9], the model ends constraints reduced to a fixed constraint. The model is shown in Fig 1 . 


\section{Nonlinear finite element method}

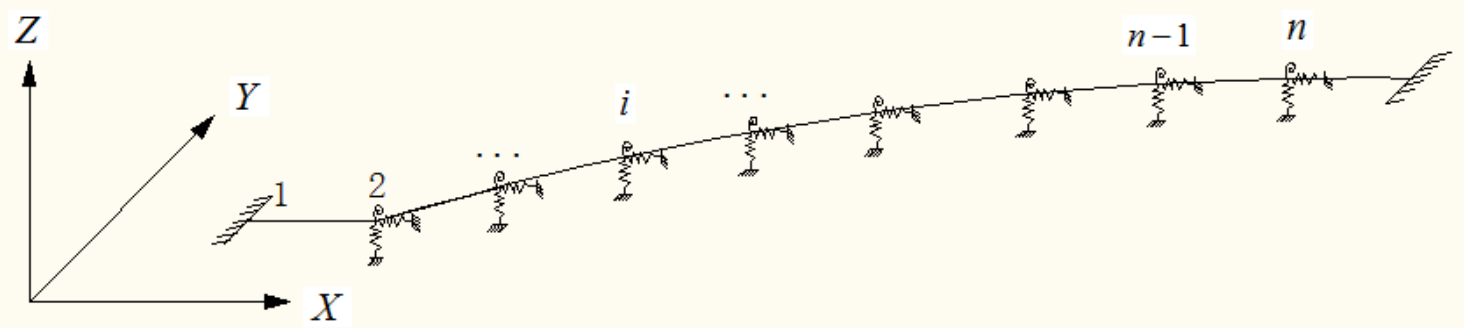

Fig.1 Calculation Model

The total potential energy of The unit beam

$$
\prod=\int_{V}\{\varepsilon\}^{T}\{\sigma\} d v-\{d\}^{T}\{p\}
$$

$\{\varepsilon\}$ is the strain matrix, $\{\sigma\}$ is the stress matrix, $\{d\}$ is the displacement matrix and $\{p\}$ is the load matrix.

$$
\{d\}=\left[\begin{array}{llllllllllll}
u_{i} & v_{i} & w_{i} & \varphi_{i} & \varphi_{y i} & \varphi_{z i} & u_{j} & v_{j} & w_{j} & \varphi_{j} & \varphi_{y j} & \varphi_{z j}
\end{array}\right]^{T}
$$

That is, each beam element has 12 degrees of freedom.

The axial strain and shear strain of the space beam element are:

$$
\varepsilon_{x}=\frac{d u}{d x}+\frac{1}{2}\left[\left(\frac{d u}{d x}\right)^{2}+\left(\frac{d v}{d x}\right)^{2}+\left(\frac{d w}{d x}\right)^{2}\right]-y \frac{d^{2} v}{d x^{2}}+z \frac{d^{2} w}{d x^{2}}
$$

$\gamma=\rho \frac{d \varphi}{d x}$

Strain matrix is expressed as:

$$
\{\varepsilon\}=\left[\begin{array}{c}
\frac{d u}{d x} \\
-y \frac{d^{2} v}{d x^{2}} \\
z \frac{d^{2} w}{d x^{2}} \\
\rho \frac{d \varphi}{d x}
\end{array}\right]+\left[\begin{array}{c}
\frac{1}{2}\left[\left(\frac{d u}{d x}\right)^{2}+\left(\frac{d v}{d x}\right)^{2}+\left(\frac{d w}{d x}\right)^{2}\right] \\
0 \\
0 \\
0
\end{array}\right]
$$

According to potential energy principle, then:

$$
\int_{V} \delta\{\varepsilon\}^{T}\{\sigma\} d V-\delta\{d\}^{T}\{p\}=0
$$


The displacement and strain can be expressed by Eq.(7) and brought it into (6)finally get Eq.(8):

$$
\begin{aligned}
& \delta\{\varepsilon\}=[B] \delta\{d\} \\
& \int_{V}[B]^{T}\{\delta\} d V=\{p\}
\end{aligned}
$$

For linear problems, view $[B]$ as constant, and for nonlinear problems , $[B]$ is displacement-dependent variable. For spatial beam elements, it is decomposed into four sub blocks as follows:

$$
[B]=\left[B_{0}\right]+\left[B_{N}^{U}\right]+\left[B_{N}^{V}\right]+\left[B_{N}^{W}\right]
$$

The right side of the equation is the linear strain matrix and the nonlinear strain matrix caused by the vertical, horizontal and vertical. Assuming a large displacement small deformation conditions, the stress and strain are linear, then:

$$
\{\delta\}=[D]\{\varepsilon\}
$$

$[D]$ as the material elastic matrix, the expression is:

$$
[D]=\left[\begin{array}{cccc}
E & 0 & 0 & 0 \\
0 & E & 0 & 0 \\
0 & 0 & E & 0 \\
0 & 0 & 0 & G
\end{array}\right]
$$

Eq.(9) and Eq.(10), the tangent stiffness matrix can be deduced. The derivation process is described in detail in [18], and is not described in this paper.

$$
\left[K_{T}\right]=\left[K_{0}\right]+\left[K_{d}\right]+\left[K_{\delta}\right]
$$

$\left[K_{T}\right]$ is the tangent stiffness matrix. $\left[K_{d}\right]$ is a large displacement matrix which includes the effects of lateral displacement, vertical displacement, lateral displacement and vertical displacement coupling, longitudinal displacement and displacement coupling. $\left[K_{\delta}\right]$ is large displacement matrix.

Using Newton-Raphson method, the iterative formula is:

$$
\left\{\begin{array}{l}
{\left[K_{T}\right]_{m}\left\{\Delta \delta_{m+1}\right\}=\left\{\Delta p_{m+1}\right\}} \\
\left\{\delta_{m+1}\right\}=\left\{\Delta \delta_{m}\right\}+\left\{\Delta \delta_{m+1}\right\}
\end{array}\right.
$$

The nonlinear finite element solution procedure of the jointless track is as follows: 
1) measuring the line is not smooth, according to the measured rod end initial displacement assignment;

2) The displacement initial value of the structure node is obtained by linear analysis;

3) Construct the tangent stiffness matrix of each bar in the local coordinate system of the bar, and calculate the nodal force of the element according to (8).

4) The tangent stiffness matrix of each bar and the nodal force steering structure coordinate system of the element;

5) group just generated the structure of the overall tangent stiffness matrix and node force vector;

6) calculate the balance force;

7) Calculate the displacement increment as follows:

$$
K_{T} \Delta \Delta=\Delta R
$$

8) adding the displacement increment to the structural displacement vector;

9) Convergence judgment, if not meet the requirements of the return to step 3), convergence conditions are as follows:

$$
\begin{aligned}
& N=\sqrt{\Delta^{T} \Delta} \\
& \frac{N^{i}-N^{i-1}}{N^{i}} \leq e
\end{aligned}
$$

\section{Deformation Analysis of Jointless Track under Vertical Settlement}

This model only analyzes the deformation of long rail under the axial temperature load, that is, take the load vector as shown in Eq.(17), the spring element stiffness matrix of the spring is shown in Eq.(18). Spring is used to express the elastic restraint of track bed, and a set of spring is applied on each tie-down node, the elastic coefficient of $K_{b x}, K_{b y}, K_{b \theta}$ is respectively.

$$
F_{\Delta t}=\left\{\begin{array}{llllllllllll}
E A \alpha \Delta t & 0 & 0 & 0 & 0 & 0 & -E A \alpha \Delta t & 0 & 0 & 0 & 0 & 0
\end{array}\right\}^{T}
$$




$$
\begin{aligned}
& K_{s p r i n g}^{e}=\left\{\begin{array}{cccccccccccc}
K_{f x} & 0 & 0 & 0 & 0 & 0 & -K_{f x} & 0 & 0 & 0 & 0 & 0 \\
0 & K_{f y} & 0 & 0 & 0 & 0 & 0 & -K_{f y} & 0 & 0 & 0 & 0 \\
0 & 0 & 0 & 0 & 0 & 0 & 0 & 0 & 0 & 0 & 0 & 0 \\
0 & 0 & 0 & K_{f s} & 0 & 0 & 0 & 0 & 0 & -K_{f s} & 0 & 0 \\
0 & 0 & 0 & 0 & 0 & 0 & 0 & 0 & 0 & 0 & 0 & 0 \\
0 & 0 & 0 & 0 & 0 & 0 & 0 & 0 & 0 & 0 & 0 & 0 \\
K_{f x} & 0 & 0 & 0 & 0 & 0 & -K_{f x} & 0 & 0 & 0 & 0 & 0 \\
0 & K_{f y} & 0 & 0 & 0 & 0 & 0 & -K_{f y} & 0 & 0 & 0 & 0 \\
0 & 0 & 0 & 0 & 0 & 0 & 0 & 0 & 0 & 0 & 0 & 0 \\
0 & 0 & 0 & K_{f s} & 0 & 0 & 0 & 0 & 0 & -K_{f s} & 0 & 0 \\
0 & 0 & 0 & 0 & 0 & 0 & 0 & 0 & 0 & 0 & 0 & 0 \\
0 & 0 & 0 & 0 & 0 & 0 & 0 & 0 & 0 & 0 & 0 & 0
\end{array}\right\} \\
& K_{f s}=2.933 \times 10^{3} s^{-\frac{1}{2}} \\
& K_{f y}=5.0 \times 10^{4} N / c m \\
& K_{f x}=\frac{10000}{x}
\end{aligned}
$$

It is assumed that the longitudinal resistance of the track bed is linearly related to the longitudinal displacement,

$$
K_{b x}=\frac{5000}{x}
$$

In this relation, it is assumed that the longitudinal resistance of the track bed of a single sleeper of the concrete sleeper line is a constant of $10,000 \mathrm{~N}$.

It is assumed that the lateral resistance of the track bed is linearly related to the lateral displacement.

$$
Q=4 K_{b y} y
$$

The lateral elastic modulus of the track bed is obtained by solving the slope of the relationship between lateral resistance and lateral displacement of the track bed:

$$
K_{b y}=\frac{Q^{\prime}}{4}=\frac{q^{\prime}}{4} a=\frac{a}{4}\left(-B Z y^{Z-1}+\frac{C}{N} y^{\frac{1}{N}-1}\right)
$$

$\mathrm{B}, \mathrm{C}, \mathrm{Z}, \mathrm{N}$ for the drag coefficient, a for the sleeper spacing. But the change of the bed resistance value will change with the vertical displacement of the track bed. Therefore, the initial value of the bed resistance value should be modified according to the vertical displacement when 
solving the longitudinal and lateral elastic modulus of the track bed in a certain state. At home and abroad on the orbital float after the bed resistance calculation is based on the relationship between sleeper reaction force and the sleep weight, when the sleeper reaction force is greater than the sleeper weight, the weight of sleepers and track bed friction resistance failure, should be in the resistance And the friction between the sleepers and track bed has not been completely lost. Therefore, the modified bed resistance should be calculated as follows:

$$
Q_{s}=\frac{2 \times\left(\frac{2}{3} \times H-W\right)}{B+2 \times\left(\frac{2}{3} \times H\right)} Q_{0}
$$

$W$ is the vertical displacement of the node, $B$ is the sleeper width, $H$ is the middle of the sleeper interface height. It is calculated that the bed loss calculated by the formula is $7.5 \%$ higher than the measured value in the literature [10]. The calculation is in good agreement with the data of different sleeper and buried depth of different sleepers , and need further verification.

$$
K_{b \oplus}=1.0 \times 10^{6} \mathrm{~N} / \mathrm{cm}
$$

In this paper, $60 \mathrm{Kg} / \mathrm{m}$ rails, spring type II fasteners and concrete type II sleeper are used in this model, with 1840 per kilometer, and the initial state of the track bed is compacted. The bed resistance is described by the spring constraint, and the longitudinal and lateral spring restraints are imposed on each rail joint. The lateral resistance of the track bed is taken as the slope of the corresponding curve of the lateral resistance-displacement curve. As shown in Fig. 2, when the rail temperature is raised by $50{ }^{\circ} \mathrm{C}$, the displacement of the long rail is shifted. The displacement of the rail is bounded by the middle node. The displacement of the front section is reverse to the Y axis. The trend of change is parabolic. The displacement peak is $1.8681 \mathrm{~mm}$, and the long rail is distorted. Under the conditions shown in Figure 2, the rail only by the local coordinate system under the axial temperature force, its vertical displacement. In view of the continuity of the long rail, the vertical displacement of the single node or nodes will drive the other nodes to produce vertical displacement. In the following, single point and double point ups and downs and double point cross ups and downs will be taken separately. Displacement.

As shown in Figure 3, when the No. 2 node settlement, will lead to the rest of the rail settlement. The displacement of each node of the rail is negative, which is reverse to the Z-axis of the global coordinate system. The vertical displacement of nodes 1 to 5 gradually increases, and the displacement peak is generated at node 5, and then the displacement gradually returns The displacement trend of the rail under the condition of floating $2 \mathrm{~mm}$ is the same as the subsidence, and the direction is opposite.

When the two nodes in the same direction of displacement occurs, the vertical displacement of the rail shown in Figure 4. Take the model 2, 3 nodes, to produce the overall coordinate system $\mathrm{Z}$-axis with the reverse $2 \mathrm{~mm}$ displacement, analog circuit settlement. When the No. 2 and No. 2 nodes sink $2 \mathrm{~mm}$, the remaining nodes of the long rail are Z-axis reverse displacement, the overall rail sink. 2 and 3 nodes go up $2 \mathrm{~mm}$ and sink the same situation, the opposite direction. Unlike the case shown in Fig. 3, when the displacement of nodes 2 and 3 occurs in the same direction, the displacement of nodes 2 and 3 causes the rail displacement to occur at nodes 2 and 3. 
As shown in Figure 5, when the No. 2 node settlement 2mm, 3 nodes floating $2 \mathrm{~mm}, 4$ to 12 node displacement is positive, the overall trend of the line was floating. The displacement changes in a parabolic form, the direction of parabolic development and the last point of the same direction of displacement, the peak value of more than $8 \mathrm{~mm}$, at the 6th node, the middle of the long rail, for all vertical settlement in this case the maximum. When the No. 2 node floating $2 \mathrm{~mm}, 3$ nodes sinking $2 \mathrm{~mm}$, the change is the same with the previous situation, in the opposite direction.

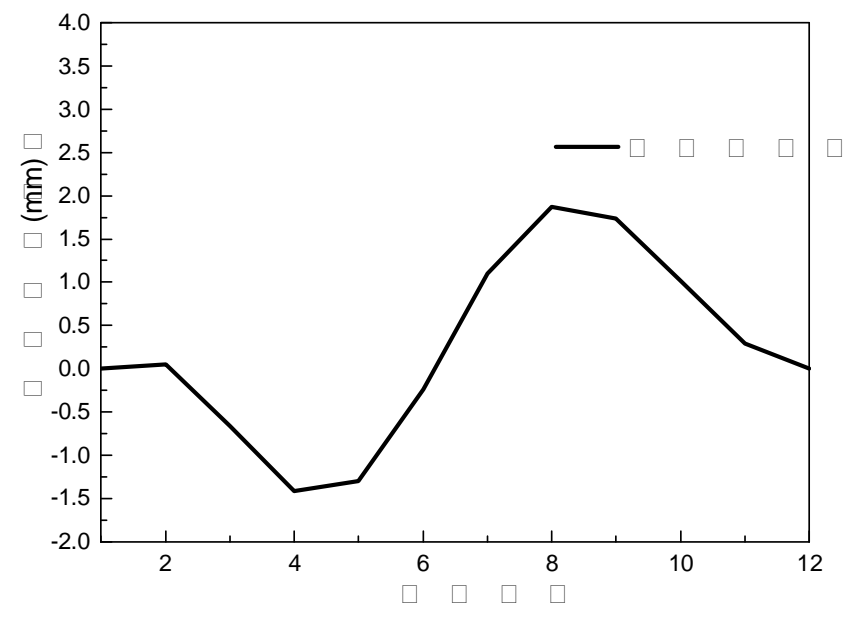

Fig.2 Rail lateral displacement under heating conditions

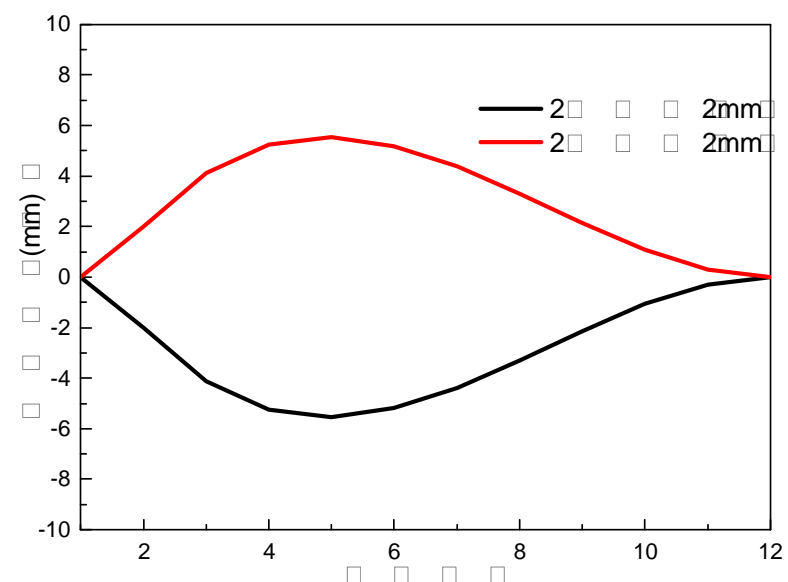

Fig.3 2 Rail vertical displacement at node 2 of forced displacement conditions

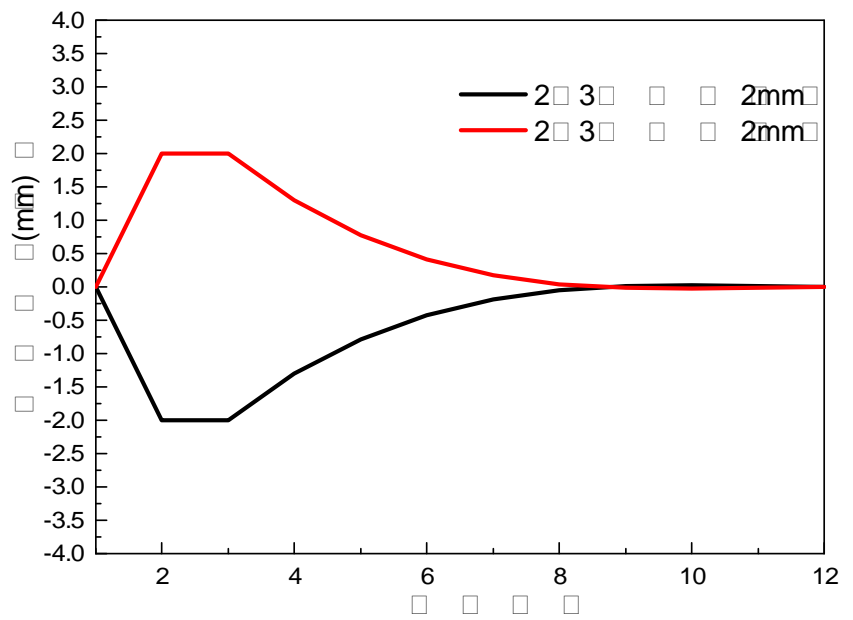

Fig.4 2,3 node rail vertical displacement under the same displacement conditions 


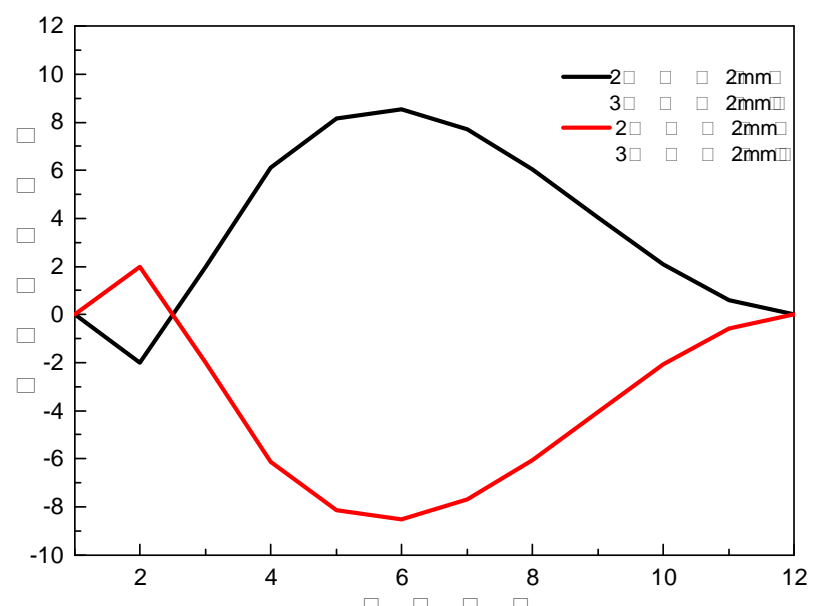

Fig.5 2,3 node rail vertical displacement under the cross displacement conditions

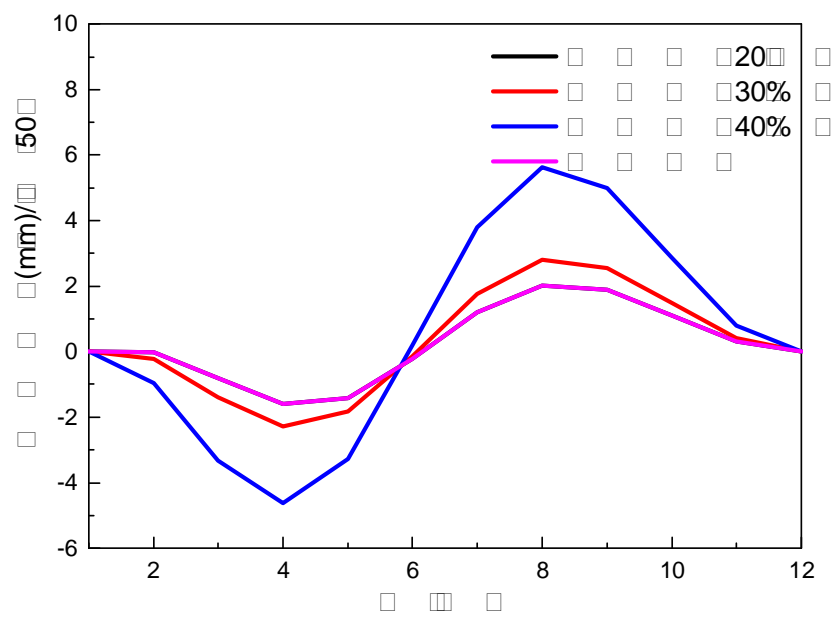

Fig.6 Rail lateral displacement curve of subgrade settlement conditions

To sum up, the vertical displacement of the rail joints is the most disadvantageous condition, the longitudinal displacement of the middle part of the long rail is the biggest, and decreases toward both ends. The transverse resistance-displacement curve of the track bed is analyzed, and the lateral resistance of the track bed is lost about $40 \%$ when the rail joint is $3.5 \mathrm{~mm}$. Combined with the rail vertical displacement of the most unfavorable conditions, take the middle part of the long rail for the maximum amount of float to the two ends of the law was a quadratic decline. As shown in Fig. 6 , the lateral displacement of the long rail increases with the decrease of lateral resistance of track bed, and the displacement trend is consistent with Fig. In a certain range, the increase of transverse displacement of long rail is linear with the loss of track bed resistance. When the resistance of track bed is lost by $40 \%$, the lateral displacement of long rail suddenly increases and the maximum displacement is $5.6292 \mathrm{~mm}$.

\section{Conclusions}

When the settlement or arching of the subgrade occurs, the vertical displacement of the adjacent nodes of the long rail will be the same as the deformation direction of the subgrade, and the displacement of the rail will be small under the uniform settlement of the subgrade.

When the embankment settlement and the arched alternation, the rail displacement value increased significantly, the displacement direction and deformation direction of the final roadbed.

Determine the vertical displacement value of each node according to the settlement and settlement range of the roadbed, and give the range and range of the loss of the bed resistance. The 
transverse displacement of the rail is approximately linear with the resistance loss of the track bed at the given rail temperature. The greater the resistance loss of the track bed, the greater the transverse displacement value of the rail. When the loss of the track bed exceeds the critical value, the lateral displacement of the rail increases significantly, showing instability trend.

\section{References}

[1]Zhang Xiangming,Gao Liang,Cui Rixin,Zeng Zhiping,Chen Xianmai.Research on the technology of design,laying and maintenance for CWR in permafrost regions[J].Journal of Railway Science and Engineering,2016,13(2):257 - 262 .

[2]Zhang Xiangming,Gao Liang,Cui Rixin.Research on Monitoring Technology for CWR of Qinghai - Tibet Railway in Permafrost Region[J]. Railway Engineering,2016,(4):114 - 117 .

[3]Zhang Xiangming,Gao Liang,Cui Rixin,Zeng Zhiping,Chen Xianmai.Experimental Research on the State Index of CWR in Golmud - Lhasa Section of Qinghai - Tibet Railway[J].Journal of Railway Engineering Society,2016,(5):26 - 31 .

[4]Quan Shunxi,Zhang Yao,Wang Ping.The Influence of Beam-End Lateral Expansion of Steel Truss Girder on Stability of CWR Track[J].ICTE,2011:1690-1695 .

[5]Wei Xiankui,Wang Ping,Pang Ling,Chen Rong.One strengthening scheme of stability of small radiuscurve continuous welded rail on bridge[J]. Journal of Railway Science and Engineering,2012, 9(4):14 - 18 .

[6]Liang Can.A Study on the Stability of Continuously Welded Rail Tracks and Relevant Effective Measures[J].Journal of Railway Engineering Society,2012,(1):26 - 29 .

[7]Zhou Yi.Seamless Line Temperature Drum up Instability Process of Finite Element Analysis. China Railway Science.1988,9(2): 12-20.

[8]Zeng Zhiping, Yu Zhiwu.Power Spectral Density Analysis of Track Irregularity of CWR Track of Qinghai-Tibet Railway[J].Logistics,2009:2294-2299.

[9]Zhao Weihua, Wang Ping,Cao Yang.Additional Temperature Forces of Continuous Welded Rail on Large-Span Steel Truss Cable-Stayed Bridge[J].ICTE,2011:1767-1773 .

[10]Lu Yaorong. The Analysis and Research of The Seamless Line Dynamic Instability Characteristics.Journal of the China Railway Society, 1993,15(3): 77-83.

[11]Gao Liang, Wan Fuguang. Inquire into Studying Method on the Dynamic Stability of the Continuously Welded Rail Track. China Railway Science, 1998,19(2): 76-82. 